\title{
The Square-Lattice Heisenberg Antiferromagnet at Very Large Correlation Lengths
}

\author{
B.B. Beard ${ }^{a}$, R.J. Birgeneau ${ }^{b}$, M. Greven ${ }^{b}$, and U.-J. Wiese ${ }^{b}$ \\ ${ }^{a}$ Departments of Physics and Mechanical Engineering, Christian Brothers University, Memphis, TN 38104 \\ ${ }^{b}$ Department of Physics, Massachusetts Institute of Technology, Cambridge, MA 02139
}

(May 22, 2018)

\begin{abstract}
The correlation length of the square-lattice spin-1/2 Heisenberg antiferromagnet is studied in the lowtemperature (asymptotic-scaling) regime. Our novel approach combines a very efficient loop cluster algorithm - operating directly in the Euclidean time continuum - with finite-size scaling. This enables us to probe correlation lengths up to $\xi \approx 350,000$ lattice spacings - more than three orders of magnitude larger than any previous study. We resolve a conundrum concerning the applicability of asymptotic-scaling formulae to experimentally- and numerically-determined correlation lengths, and arrive at a very precise determination of the low-energy observables. Our results have direct implications for the zero-temperature behavior of spin-1/2 ladders.
\end{abstract}

02.70.Lq,31.15.Kb,75.10.Jm,75.50.Ee

Soon after the discovery of high-temperature superconductivity in doped lamellar copper oxides it was found that the undoped compounds are quasi-two-dimensional (2-d) spin $S=1 / 2$ quantum antiferromagnets. A theoretical model that captures the essential features of these materials is the nearest-neighbor quantum antiferromagnetic Heisenberg model (AFHM) on a square lattice. Through experimental, numerical, and theoretical efforts much progress has been made in the understanding of these systems. In particular, detailed neutron scattering measurements of the spin-spin correlation length in the magnet $\mathrm{Sr}_{2} \mathrm{CuO}_{2} \mathrm{Cl}_{2}$ were found to be described quantitatively [1] by both high-temperature numerical results for the AFHM [2] and low-temperature theory for the renormalized classical regime of the $(2+1)$-d $O(3)$-symmetric non-linear $\sigma$-model [3,, 1 .

The ground state of the above systems shows longrange antiferromagnetic order, thus spontaneously breaking the $O(3)$ rotational symmetry to $O(2)$. The low-energy excitations are two massless bosons called magnons or spin-waves. These long-range excitations determine the dynamics at low energies. One can use chiral perturbation theory (CPT) to derive universal expressions for low-energy observables in terms of three material-specific parameters: the staggered magnetization $\mathcal{M}_{s}$, the spin-wave velocity $c$, and the spin stiffness $\rho_{s}$ [5]. Both numerical data and predictions of CPT are in apparent good agreement with experimental results for $S=1 / 2$ [1]. However, neutron scattering measurements on antiferromagnets with $S>1 / 2$ [1] 6] reveal a striking discrepancy with CPT predictions based on 3loop asymptotic scaling. It has been suggested that for $S>1 / 2$, asymptotic scaling sets in only at very low temperatures - that is, for correlation lengths much larger than those accessed experimentally and numerically [7].

In this Letter, we investigate the correlation length of the 2-d nearest-neighbor square-lattice spin-1/2 Heisenberg antiferromagnet at unprecedentedly low tempera- tures. We resolve the puzzle concerning the applicability of asymptotic scaling to experimentally- and numericallydetermined correlation lengths [1, 6 -8]. To effect this, we combine an efficient loop cluster algorithm operating in the Euclidean time continuum - hence with zero systematic error - with a finite-size scaling technique. With this powerful new approach, the infinite-volume correlation length $\xi$ is probed up to $\approx 350,000$ lattice spacings $a$. This allows us to paint a detailed picture of the variation of $\xi$ with temperature well into the asymptotic scaling regime, and to arrive at the presently most precise determination of the low-energy observables of the spin$1 / 2$ AFHM. We find that asymptotic scaling of $\xi$ with the 3-loop $\beta$-function of the 2 -d classical $O(3)$ model sets in only at about $10^{5} a$, while a 4 -loop fit describes the data already at $\xi / a \approx 100-200$, corresponding to the largest correlation lengths measured experimentally [1]. By exchanging a spatial with the Euclidean time direction, as suggested in Ref. 9, our results may be applied to spin- $1 / 2$ ladders at zero temperature.

In $\mathrm{CPT}$, the 2-d quantum spin system is described by a $(2+1)$-d $O(3)$ symmetric Euclidean field theory. At non-zero temperature $T$ the Euclidean time direction has a finite extent $1 / T$. For a system of massless particles - that is, one with an infinite zero-temperature correlation length - the non-zero temperature system appears dimensionally reduced to two dimensions, because $1 / T$ is then negligible compared to $\xi$. However, the Hohenberg-Mermin-Wagner-Coleman theorem forbids interaction among massless Goldstone bosons in two dimensions. Consistent with this, the 2-d $O(3)$ model is known to have a non-perturbatively generated mass gap. This in turn implies that the spin-waves of a 2-d quantum antiferromagnet at non-zero temperature also acquire a mass. Using perturbative renormalization group arguments, Chakravarty, Halperin, and Nelson (CHN) [3] derived the expression 


$$
\xi=0.31(4) \frac{c}{2 \pi \rho_{s}} \exp \left(\frac{2 \pi \rho_{s}}{T}\right)\left[1+\mathcal{O}\left(\frac{T}{\rho_{s}}\right)\right] .
$$

Consistent with dimensional reduction, $\xi$ is exponentially large compared to $c / T$. We note that the exponent in Eq.(11) comes from a 1-loop calculation, while the factor $c / 2 \pi \rho_{s}$ is a 2-loop result.

Hasenfratz and Niedermayer [1] averaged the $(2+1)$ $d$ field over cubic space-time volumes of size $1 / T$ in the Euclidean time direction and $c / T$ in the two spatial directions. Since at low temperatures $\xi \gg c / T$, the field is essentially constant over these blocks. The averaged field naturally lives at the block centers, which form a 2$\mathrm{d}$ lattice of spacing $a^{\prime}=c / T$ (which is different from the lattice spacing $a$ ). Hence, the effective action of the averaged field defines a 2-d lattice $O(3)$ model. Using CPT, as well as the exact mass-gap and the 3 -loop $\beta$-function of the 2-d $O(3)$ model [10], Hasenfratz and Niedermayer extended the CHN-formula to

$$
\xi=\frac{e}{8} \frac{c}{2 \pi \rho_{s}} \exp \left(\frac{2 \pi \rho_{s}}{T}\right)\left[1-\frac{T}{4 \pi \rho_{s}}+\mathcal{O}\left(\frac{T^{2}}{\rho_{s}^{2}}\right)\right],
$$

which we call the $\mathrm{CH}_{2} \mathrm{~N}_{2}$-formula. This equation is valid at low temperatures, that is, for large correlation lengths. When the correlation length is correctly described by Eq.(2), it scales asymptotically with the 3-loop $\beta$-function of the 2-d $O(3)$ model. The undetermined $\mathcal{O}\left(T^{2} / \rho_{s}^{2}\right)$ term represents a 4 -loop effect.

In relativistic quantum field theory the lattice spacing serves as an ultraviolet cut-off that is ultimately removed. The question of asymptotic scaling is thus unphysical because it involves the bare coupling constant. In the 2-d AFHM, however, the lattice spacing of the induced classical model is $a^{\prime}=c / T$, where $T$ is the physical temperature. Hence, the question of asymptotic scaling becomes physical. A priori, it is unclear for what values of $\xi$ one should expect asymptotic scaling for the effective 2-d lattice action.

This raises the important question: for what $\xi$ does the $\mathrm{CH}_{2} \mathrm{~N}_{2}$-formula work correctly? We address this issue for the AFHM, defined by the Hamiltonian

$$
H=J \sum_{x, \mu} \vec{S}_{x} \cdot \vec{S}_{x+\hat{\mu}}
$$

where $J>0$ is the antiferromagnetic coupling, $\vec{S}_{x}$ is a quantum spin- $1 / 2$ operator located at point $x$ of a square lattice with spacing $a$, and $\hat{\mu}$ is the unit vector in the $\mu$ direction. Previously, comparisons of Monte Carlo calculations of the staggered and uniform susceptibilities $\left(\chi_{s}\right.$ and $\chi_{u}$ ) with CPT predictions yielded $c=1.68(1) \mathrm{Ja}$, $\rho_{s}=0.186(4) J$, and $\mathcal{M}_{s}=0.3083(2) / a^{2}[11,12]$. The calculation of Ref. [11] indicates that CPT results for $\chi_{s}$ and $\chi_{u}$ of the same kind as Eq.(2) - but with known $\mathcal{O}\left(T^{2} / \rho_{s}^{2}\right)$ terms - work very well for the AFHM for $T \leq 0.2 J$. Hence, one expects that asymptotic scaling at the 4-loop level sets in at about the same temperature. At $T=0.2 \mathrm{~J}$ the $\mathrm{CH}_{2} \mathrm{~N}_{2}$-formula predicts $\xi / a \approx 150$. To avoid finitesize effects in numerical simulations, one must simulate lattices with a spatial extent at least $L \approx 6 \xi$. Even with the best algorithms available 13, 11, 12, working on lattices starting with $L^{2}=900^{2}$ is extremely timeconsuming.

How then can we investigate $\xi$ in the low-temperature regime? We make use of finite-size effects instead of trying to avoid them. Finite-size scaling methods were used by Kim [14] and by Caracciolo et al. [15] to test asymptotic scaling of the correlation length with the bare coupling constant in the classical 2-d lattice $O(3)$ model. To investigate $\xi$ in the AFHM, we apply the technique of Ref. [15, in which renormalization group methods are used to justify the search for a universal scaling function

$$
\xi(2 L) / \xi(L)=F(\xi(L) / L) .
$$

Carracciolo et al. 15] showed that their data indeed collapse to a single scaling function $F(\xi(L) / L)$. They also showed that iteration of Eq.(俄) yields rapid convergence to $\xi$, the correlation length of the infinite system.

The universal function $F$ for the classical 2-d $O(3)$ model was determined very precisely in Ref. [15]. Since at low temperatures the quantum model reduces to a classical 2-d lattice $O(3)$ model, one can use the same function to deduce $\xi$ from $\xi(L)$ data. We verified that indeed the same scaling function works for the Heisenberg model with measurements of $\xi(L)$ for lattices of successively larger size. This calculation yields very good, though not exact, agreement with $F(\xi(L) / L)$. We incorporated a tiny correction to the scaling function to account for these violations at each stage of the iteration. We found that the correlation length so determined was insensitive to the form of the scaling violation, for several different fitted functional forms.

We emphasize that Eq.(4) assumes universal behavior, that is, scaling, but not asymptotic scaling. This is important, because we want to use Eq.(化) to compare with the $\mathrm{CH}_{2} \mathrm{~N}_{2}$-formula Eq.(2) without bias.

The scaling procedure is very sensitive to small changes in the finite-volume correlation length $\xi(L)$, that is, a small error in $\xi(L)$ can lead to large uncertainties in the infinite-volume $\xi$. Hence, one needs a very accurate numerical method to determine $\xi(L)$. Fortunately, for the AFHM there exists a very efficient loop cluster algorithm [13,11, which practically eliminates auto-correlations in successive Monte Carlo configurations. The cluster algorithm also enables improved estimators which drastically reduce statistical errors. Finally, implementing the cluster algorithm in continuous Euclidean time completely eliminates the systematic error due to the Trotter-Suzuki discretization of time [12]. This continuous-time cluster algorithm (CTCA) has greatly reduced storage and computer time requirements, enabling simulation at very low temperatures. 
For $J / T=0.5,1.0, \ldots, 3.5$, we carry out simulations that measure the correlation length directly in a large volume $L \approx 6 \xi$, while for $J / T=4.0,4.5, \ldots, 12.0$, we use the finite-size scaling technique. A single-cluster version of the CTCA is used with an improved estimator for the staggered correlation function, then $\xi(L)$ is extracted from the correlation function using the secondmoment method [14 15. For the direct-measurement cases, $10^{5}$ Monte Carlo configurations are used; for the scaled-measurement cases, $4 \times 10^{5}$ are used. The numerical data for $\xi(L)$ together with the inferred infinitevolume $\xi$ are given in Table I, and are compared with experimental data and the $\mathrm{CH}_{2} \mathrm{~N}_{2}$-formula in Fig.1. The largest accessible $\xi$ is more than three orders of magnitude larger than those of any previous study [1,2, 16, 8 .

Deviations from asymptotic scaling are invisible on the semi-log scale of Fig.1. Figure 2 shows the deviation from 2-loop asymptotic scaling as a function of $T / 2 \pi \rho_{s}$. Here, the 3 -loop result of Eq.(2) is a line of slope $-1 / 2$. The quantitative agreement shown in Fig.1 between experiment and theory in the regime $2.4<J / T<5.3$ is seen to be coincidental. We find that with decreasing temperature the correlation length crosses and undershoots the 3-loop result before approaching it from below. Consequently, the deviations at intermediate and high temperatures from the $\mathrm{CH}_{2} \mathrm{~N}_{2}$-formula turn out to be relatively small. Although neutron scattering results for $\mathrm{Sr}_{2} \mathrm{CuO}_{2} \mathrm{Cl}_{2}$ cover a wide range of $\xi$ with relatively small errors [1], the deviations from 3-loop asymptotic scaling turn out to be too subtle to be discernable experimentally. This explains why the low-temperature result of Eq.(2) appears to describe the experimental data at such high temperatures.

Owing to the exponential dependence on $\rho_{s}$, the placement of the numerical data on the graph of Fig.2 is extremely sensitive to $\rho_{s}$ in the low- $T$ regime. This enables a very precise estimate of $\rho_{s}$. The correlation length data are fitted simultaneously with previously obtained results for $\chi_{s}$ and $\chi_{u}$ in cubical [11] and cylindrical [12] spacetime geometries, resulting in

$$
c=1.657(2) J a, \rho_{s}=0.1800(5) J, \mathcal{M}_{s}=0.30797(3) / a^{2} \text {. }
$$

This is in very good agreement with results of an expansion around the Ising limit, which gives $c=1.654(11) \mathrm{Ja}$, $\rho_{s}=0.182(5) J$, and $\mathcal{M}_{s}=0.307(1) / a^{2}$ [17].

In order to fit the correlation length data down to $\xi / a \approx 100$, it was necessary to include the $\mathcal{O}\left(T^{2} / \rho_{s}^{2}\right)$ 4-loop term - not determined in Ref. [10] - with a fitted coefficient $C_{2}$. We find $C_{2}=-0.75(5)$ if the fit is restricted to quadratic order and to $J / T>2.8$. Higherorder fits (including $C_{3}, C_{4}, \ldots$ ) shift the value determined for $C_{2}$, although it remains $\mathcal{O}(1)$ and stable. A previous study also found that quadratic-order terms are needed to match the precision of the $\chi_{s}$ and $\chi_{u}$ measured with the CTCA [12]. Thus, it should come as no surprise that asymptotic scaling at the 3-loop level of the $\mathrm{CH}_{2} \mathrm{~N}_{2}$-formula sets in at rather large correlation lengths of $\xi \approx 10^{5} a$. The same is true for the classical 2-d $O(3)$ model with the standard lattice action 15.

We also carried out an additional independent fit for the prefactor in the $\mathrm{CH}_{2} \mathrm{~N}_{2}$-formula. That is, we multiplied Eq.(2) by an extra fitted parameter $A$, and found $A=1.02(2)$ (using the 4-loop form for the $\beta$-function), in excellent agreement with Eq.(2). This gives added confidence both in our scaling procedure and in the correctness of the exact massgap.

A large discrepancy between the $\mathrm{CH}_{2} \mathrm{~N}_{2}$-formula and experimental data has been discovered for systems with $S>1 / 2$ [1],6]. A likely explanation of this discrepancy is that 3-loop asymptotic scaling again sets in only at very small temperatures. In fact, the existence of a crossover from high- to low-temperature behavior was suggested in Ref. [7]. Although the asymptotic region is inaccessible to experiments, we have demonstrated that it is possible to investigate it using the cluster algorithm in combination with finite-size scaling. A similar study for $S>1 / 2$ is presently in progress.

Recently, it was pointed out that the $\mathrm{CH}_{2} \mathrm{~N}_{2}$-formula can also be applied to the correlation length at $T=0$ of even-width Heisenberg spin-ladders [9]. These ladders comprise an even number $n$ of coupled $S=1 / 2$ chains with periodic boundary conditions. This mapping exchanges one spatial direction with the Euclidean time direction, so the time extent plays the role of $n$. The 3 -loop $\beta$-function is the same for the square-lattice and the ladder. In contrast, the undetermined higher-order terms are influenced by the distinction that space is a lattice, whereas Euclidean time is continuous. Hence, the $\mathcal{O}\left(T^{2} / \rho_{s}^{2}\right)$ terms are expected to be different for the square-lattice and the spin-ladders. Early density-matrix renormalization group calculations of the spin gap of even-width ladders were reported in Ref. [18. The correlation length of spin-1/2 ladders was studied numerically for $n \leq 6$ [16,19]. We see in Fig.2 that 3-loop asymptotic scaling sets in only at $T \approx 0.13 J$. We thus expect the 3-loop $\mathrm{CH}_{2} \mathrm{~N}_{2}$-formula to work quantitatively only for ladders with widths greater than $n=c / T \approx 12$. It was already realized in Ref. [19] that the 3-loop result is insufficient to describe the numerical data for $n \leq 6$. A 4-loop form should work for $n \geq 6$.

In conclusion, we have combined a powerful and accurate quantum Monte Carlo technique with finite-size scaling. This has made possible the determination of the correlation length of the $S=1 / 2$ nearest-neighbor square-lattice antiferromagnetic Heisenberg model at unprecedentedly low temperatures. Our study resolves the conundrum concerning the applicability of the 3-loop asymptotic scaling description for $S=1 / 2$, and it points the way toward a quantitative resolution of this issue for $S>1 / 2$. Our results also have direct implications for the low-temperature properties of $S=1 / 2$ ladders. 
We are indebted to A. Ferrando and F. Niedermayer for very interesting discussions. We also thank A. Pelissetto for providing us with the universal finite-size-scaling function $F$. This work was supported by the NSF under Grant No. DMR 97-04532, the International Joint Research Program of NEDO (New Energy Development Organization) International Joint Research Grant, and the DOE under cooperative research agreement number DE-FC02-94ER40818. U.-J.W. is also supported by an A.P. Sloan fellowship.

[1] M. Greven et al., Phys. Rev. Lett. 72, 1096 (1994); Z. Phys. B 96, 465 (1995).

[2] M.S. Makivić and H.-Q. Ding, Phys. Rev. B 43, 3562 (1991).

[3] S. Chakravarty, B.I. Halperin, and D.R. Nelson, Phys. Rev. B 39, 2344 (1989).

[4] P. Hasenfratz and F. Niedermayer, Phys. Lett. B 268, 231 (1991).

[5] P. Hasenfratz and H. Leutwyler, Nucl. Phys. B 343, 241 (1990); P. Hasenfratz and F. Niedermayer, Z. Phys. B 92, 91 (1993).

[6] K. Nakajima et al., Z. Phys. B 96, 479 (1995); Y.S. Lee et al., to be published.

[7] N. Elstner et al., Phys. Rev. Lett. 75, 938 (1995).

[8] J.-K. Kim, D.P. Landau, and M. Troyer, Phys. Rev. Lett. 79, 1583 (1997).

[9] S. Chakravarty, Phys. Rev. Lett. 77, 4446 (1996).

[10] P. Hasenfratz, M. Maggiore, and F. Niedermayer, Phys. Lett. B 245, 522 (1990); P. Hasenfratz and F. Niedermayer, Phys. Lett. B 245, 529 (1990).

[11] U.-J. Wiese and H.-P. Ying, Z. Phys. B 93, 147 (1994).

[12] B.B. Beard and U.-J. Wiese, Phys. Rev. Lett. 77, 5130 (1996).

[13] H.G. Evertz, G. Lana, and M. Marcu, Phys. Rev. Lett. 70, 875 (1993).

[14] J. K. Kim, Phys. Rev. Lett. 70, 1735 (1993); Phys. Rev. D 50, 4663 (1994).

[15] S. Caracciolo et al., Phys. Rev. Lett. 75, 1891 (1995).

[16] M. Greven, U.-J. Wiese, and R.J. Birgeneau, Phys. Rev. Lett. 77, 1865 (1996).

[17] Z. Weihong, J. Oitmaa, and C.J. Hamer, Phys. Rev. B 43, 8321 (1991); C. Hamer, Z. Weihong, and J. Oitmaa, Phys. Rev. B 50, 6877 (1994).

[18] S.R. White, R.M. Noack, and D.J. Scalapino, Phys. Rev. Lett. 73, 886 (1994).

[19] O.F. Syljuåsen, S. Chakravarty, and M. Greven, Phys. Rev. Lett. 78, 4115 (1997).

TABLE I. Finite- and infinite-volume correlation length. Infinite-volume $\xi / a$ are deduced using finite-size scaling.

\begin{tabular}{rccr}
\hline \hline$J / T$ & $L / a$ & $\xi(L) / a$ & $\xi / a \equiv \xi(\infty) / a$ \\
\hline 0.5 & 20 & $0.481(1)$ & $0.481(1)$ \\
1.0 & 80 & $0.973(2)$ & $0.973(2)$
\end{tabular}

\begin{tabular}{rccc}
1.5 & 80 & $1.818(4)$ & $1.818(4)$ \\
2.0 & 80 & $3.351(8)$ & $3.351(8)$ \\
2.5 & 80 & $6.23(1)$ & $6.23(1)$ \\
3.0 & 160 & $11.60(3)$ & $11.60(3)$ \\
3.5 & 160 & $21.23(2)$ & $21.25(2)$ \\
4.0 & 80 & $31.3(1)$ & $39.4(2)$ \\
4.5 & 80 & $39.0(1)$ & $69.9(7)$ \\
5.0 & 80 & $44.6(1)$ & $126(2)$ \\
6.0 & 80 & $52.5(1)$ & $403(6)$ \\
7.0 & 80 & $58.8(1)$ & $1275(27)$ \\
8.0 & 80 & $64.2(1)$ & $3960(120)$ \\
10.0 & 80 & $73.5(1)$ & $38460(1010)$ \\
12.0 & 160 & $159.0(2)$ & $352000(10500)$ \\
\hline \hline
\end{tabular}


FIG. 1. Experimental [1] (filled circles) and computed values (open circles; errors are much smaller than symbol size) of the staggered-spin correlation length. The solid line is the 3-loop $\mathrm{CH}_{2} \mathrm{~N}_{2}$-formula, Eq.(2), with $\rho_{s}=0.1800$.

FIG. 2. Analysis of the correlation length as the asymptotic scaling regime is approached. To remove the exponential inverse-temperature dependence, $\xi$ is divided by the 2-loop formula, using the best-fit value $\rho_{s}=0.1800(5)$. The 3-loop result (straight line) is accurate only for $\xi / a \approx 10^{5}$, while the 4-loop regime (parabola) begins at roughly $\xi / a \approx 10^{2}$. 


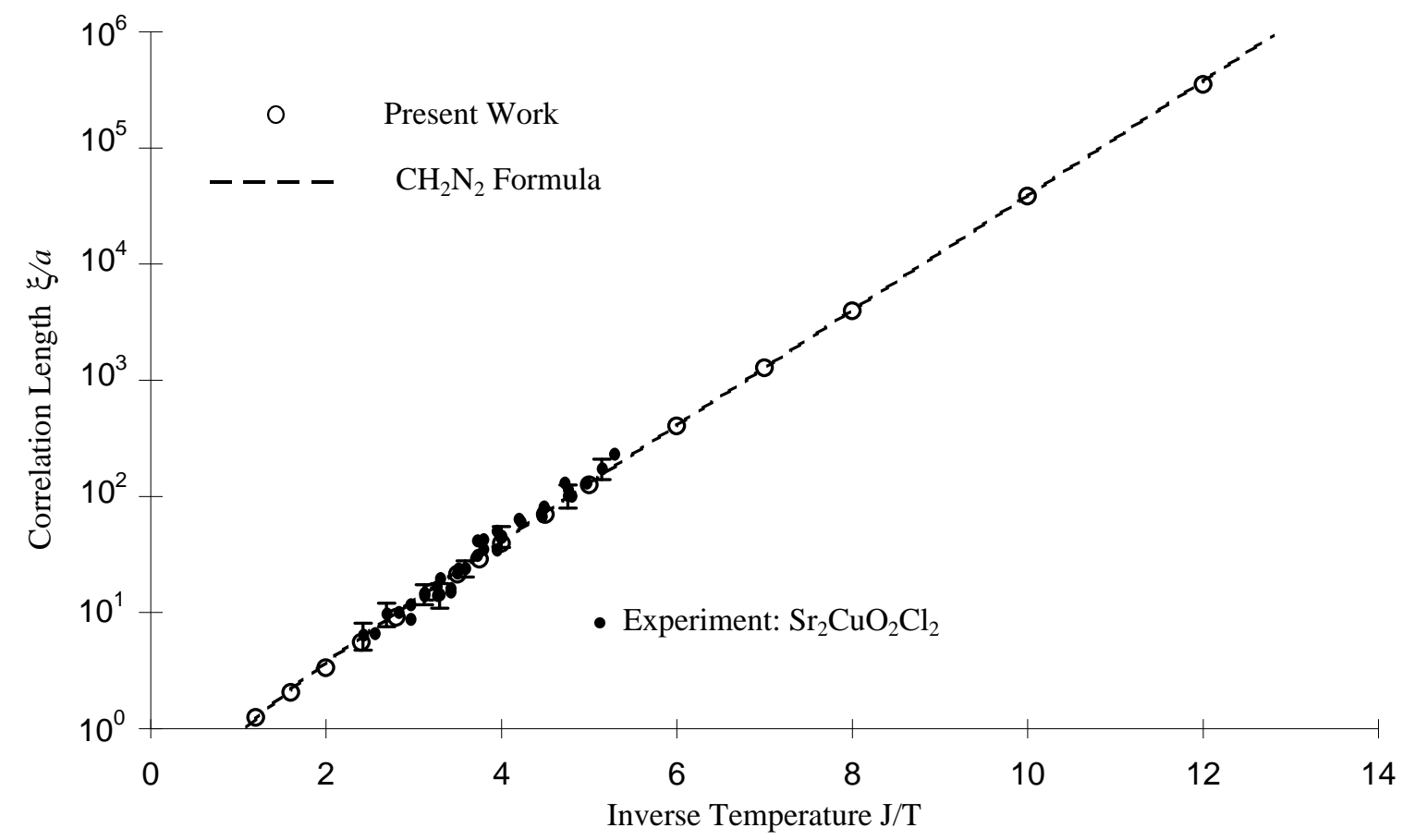

FIG. 1. Experimental [1] (filled circles) and computed values (open circles; errors are much smaller than symbol size) of the staggered-spin correlation length. The dashed line is the 3-loop $\mathrm{CH}_{2} \mathrm{~N}_{2}$ formula, Eq.(2), with $\rho_{\mathrm{s}}=0.1800$.

This is Figure 1 for B.B. Beard, R.J. Birgeneau, M. Greven, and U.-J. Wiese, "The Square Lattice Heisenberg Antiferromagnet at Very Large Correlation Lengths" 


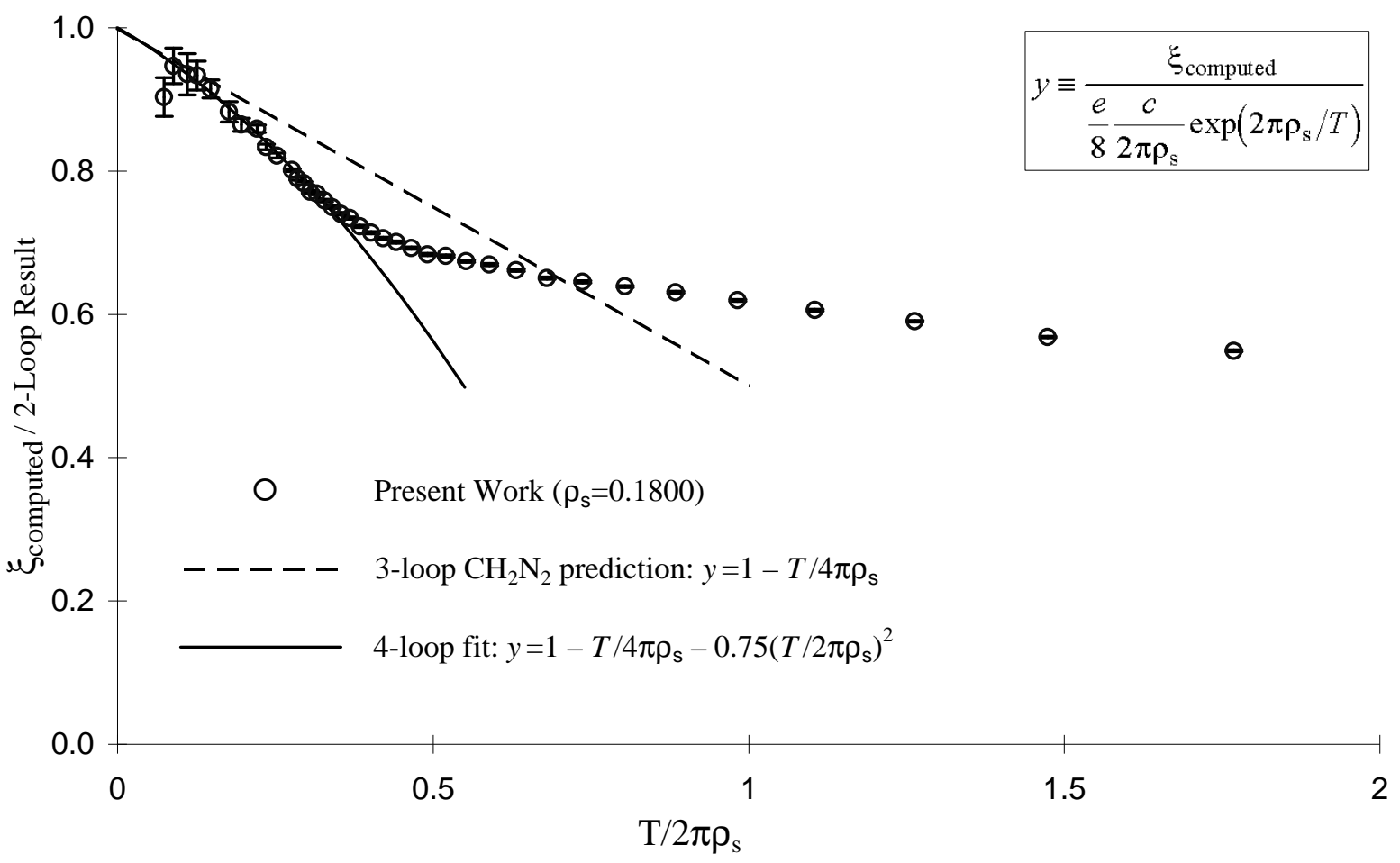

FIG. 2. Analysis of the correlation length as the asymptotic scaling regime is approached. To remove the exponential inverse-temperature dependence, $\xi$ is divided by the 2-loop formula, using the best-fit value $\rho_{\mathrm{s}}=0.1800(5)$. The 3-loop result (straight line) is accurate only for $\xi / a \approx 10^{5}$, while the 4-loop regime (parabola) begins at roughly $\xi / a \approx 10^{2}$.

This is Figure 2 for B.B. Beard, R.J. Birgeneau, M. Greven, and U.-J. Wiese, "The Square Lattice Heisenberg Antiferromagnet at Very Large Correlation Lengths" 